REST Journal on Emerging trends in Modelling and Manufacturing
Vol: 6(4), 2020
REST Publisher
ISSN: 2455-4537

Website: www.restpublisher.com/journals/jemm

\title{
A Study on Polybutylene Succinate Matrix Based Fiber Reinforced Composite
}

I Bharathwaraj, J Nalin Raj, KA. Pradheep, M.P. Jenarthanan,

School of Mechanical Engineering, SASTRA Deemed University, Thanjavur-India

jenarthanan@mech.sastra.edu

\begin{abstract}
Polybutylene succinate (PBS) (sometimes written polytetramethylene succinate) is a thermoplastic polymer resin of the polyester family. PBS is a biodegradable aliphatic polyester with properties that are comparable to polypropylene. PBS is a very promising biopolymer because its mechanical properties are comparable with those of widely used high density polyethylene and isotactic polypropylene. Like polylactic acid (PLA), it fully decomposes into biomass, $\mathrm{CO} 2$ and $\mathrm{H} 2 \mathrm{O}$ and thus can be disposed of together with other organic waste. they Polybutylene succinate are many type of test in Tensile strength test, Flexural test, scanning electron microscopy test, Impact strength test.
\end{abstract}

\section{Tensile strength}

[1] Comparison of tensile strength of RHF (rice husk flour) filled PP, HDPE, and PBS bio composites. PP-RHF and HDPERHF (rice husk flour). Weight density $10 \%$ ( filler load) of pbs-rhf (rice husk flour). Has 30\% of tensile strength, 20 $\%$ ( filler load) of pbs-rhf (rice husk flour). has $28 \%$ of tensile strength, $30 \%$ ( filler load) of pbs-rhf has $25 \%$ of tensile strength, $40 \%$ ( filler load) of pbs-rhs (rice husk flour). Has $20 \%$ of tensile strength. 1) $10 \%$ of PBS has $90 \%$ of rhf weight density 2)20\% of PBS has $80 \%$ of rhf weight density. 3) $30 \%$ of pbs has $70 \%$ of rhf weight density 4$) 40 \%$ of pbs has $60 \%$ of rhf weight density.[2] Coir fiber vs strength(MPa) Untreated :strength will be $139.67 .5 \mathrm{~N} 24(\mathrm{Mpa})$ treated:strength will be $218.52,5 \mathrm{~N} 48(\mathrm{Mpa})$ treated : strength will be $227.43,5 \mathrm{~N} 72(\mathrm{Mpa})$ treated : strength will be $238.26 .3 \mathrm{~N} 72(\mathrm{Mpa})$ treated : strength will be $209.21,7 \mathrm{~N} 72(\mathrm{Mpa})$ treated :strength will be 228.54 (Mpa) It is seen that at $5 \%$ alkali solution when soaking time increases from $24 \mathrm{~h}$ to $72 \mathrm{~h}$ the tensile properties of alkali-treated coir fibers increased, but they decreased beyond 72 h.[3]The tensile strength and modulus gradually increase with increasing basalt fiber contents. The basalt fibers can enhance the tensile strength of fiber composite from $31 \mathrm{MPa}$ to $46 \mathrm{MPa}$ as the basalt fiber loading are increased from $3 \mathrm{vol} \%$ to $15 \mathrm{vol} \%$. It is likely that the increase in the tensile strength at higher loadings of basalt fibers is relatively smaller than that at lower loadings at $0 \%$ vol basalt fiber content tensile strength is $31.5(\mathrm{MPa})$, tensile modulus is $28(\mathrm{MPa})$ at $5 \%$ vol basalt fiber content tensile strength is $43.5(\mathrm{MPa})$, tensile modulus is $36(\mathrm{MPa})$ at $15 \%$ vol basalt fiber content tensile strength is $45(\mathrm{MPa})$, tensile modulus is 42 (MPa). [4] At pressure (3mpa), 0\% fiber content, 37.6gpa tensile strength causes elongation of 16.5\%, at pressure (5mpa), 43.9\% fiber content, 106.8gpa tensile strength causes elongation of 10.7\%, at pressure (10mpa), $40 \%$ fiber content, $107.6 \mathrm{gpa}$ tensile strength causes elongation of $12.6 \%$, at pressure $(10 \mathrm{mpa}), 60 \%$ fiber content, $114.4 \mathrm{gpa}$ tensile strength causes elongation of 9.7\%. [5] The tensile properties of GNS/PBS composite were measured by a universal testing machine. At least three samples were tested to obtain average values. The surface electric conductivity at room temperature was measured with a ZC36 high resistance meter. Sample of PBS contain 0\%GNS (wt \%)( Graphene Nano sheet) and its tensile strength is $30.8(\mathrm{Mpa})$.Sample of PBS contain .5\% GNS (wt \%) (Graphene Nano sheet) and its tensile strength is 34.3(Mpa).Sample of PBS contain 1.0\%GNS (wt \%) (Graphene Nano sheet)and its tensile strength is 36.4(Mpa).Sample of PBS contain 2.0\%GNS(wt\%)(Graphene Nano sheet) and its tensile strength is 37.2(Mpa).[6]The Stretching vibration absorption of the ester group around $1750(1 / \mathrm{cm})$ increased for the esterified.The tensile properties of untreated and treated abaca fiber.Because of the serious influence of fiber diameter on the mechanical properties of the composite.Alk-abaca fibers exhibits slightly lower tensile strength and slightly higher modulus than untreated abaca fiber.The tenisle strength and modulus of AN-abaca were considerably lower than those of untreated abaca fiber.Tensile strength of untreated abaca is 813(34.4)Mpa and Tensile modulus is 33.6(32.4)GPa. Tensile strength of AA- abaca is 574(57.8)Mpa and Tensile modulus is 39.1(39.4) GPa. Tensile strength of BA- abaca is $806(30.4) \mathrm{Mpa}$ and Tensile modulus is 46.6(20.7)GPa. Tensile strength of Alk- abaca is 707(44.8)Mpa and Tensile modulus is $48.8(29.3) \mathrm{GPa}$. Tensile strength of untreated abaca is 333(44.3)Mpa and Tensile modulus is $25.9(44.3) \mathrm{GPa}$.

\section{Flexural test}

[7] The flexural properties of pure PBS and coir fiber/PBS biodegradable composites were measured by a three-point bending method according to JIS K7171 standard using universal testing machine Autograph AGS-1000A (Shimadzu, Kyoto, Japan). The flexural test was carried out at RT with a crosshead speed of $2 \mathrm{~mm} / \mathrm{min}$. The dimension of flexural specimens was 50 _ $25 \_1 \mathrm{~mm} 3$.The ratios between span distance and thickness of pure PBS and composite specimens was 16. Flexural specimens were chosen carefully before testing. The mean flexural properties of each composite were obtained from five successful test specimens. [8] Flexural in the thermal stability above 400_C was ascribed to the different content of lignin present in the 
polymer matrix. Figure 5 shows the flexural strength results of different fiber reinforced polymer composites. It was observed that among all the fibers, sisal and curaua fibers demonstrated a huge potential as reinforcement in PBS matrix. This behavior was attributed to Table Flexural modulus(Gpa) vs polymer composites1) For composite PBS flexural modulus is $=1.0$, For composite P-bagasse flexural modulus is $=1.75(\mathrm{Gpa})$, For composite P-coconut flexural modulus is $=1.6$, For composite Pcuraua flexural modulus is $=2(\mathrm{Gpa})$, For composite P-sisal flexural modulus is $=2.3[9]$ The flexural properties of silk/PBS bio composites were measured using a three-point bending method according to ASTM D790M-86 using a universal testing machine. The specimen dimensions were $50 \mathrm{~mm} \cdot 25 \mathrm{~mm} \cdot 2 \mathrm{~mm}$. The span-to-depth ratio was 16 . A load cell of $30 \mathrm{KN}$ was used. A crosshead speed of $0.85 \mathrm{~mm} / \mathrm{min}$ was used. The average values of flexural strength and modulus of each composite were obtained from ten test specimens. Flexural properties of the composites were measured by a Universal testing machine, Instron 3382, according to standards ASTM D 638 and ASTM D 790 respectively. System control and the data analysis were done using Blue Hill software. [10] Flexural and tensile properties of PHBV and PBS are improved by ABACA or sisal fiber reinforcement. Flexural strength and modulus increases with increasing fiber content. The PBS composite using BA-abaca showed the highest flexural strength. The flexural moduli increased, flexural strength did not increase regardless of the fiber treatment. Based on standard methods of testing the flexural properties of rigid plastic (JIS K7203), Span length was 30 $\mathrm{mm}$, and the testing speed was $10 \mathrm{~mm}$ per min. When PBS with lower flexural strength (43 Mpa) among the three kinds of biodegradable polyesters studied, is used as matrix polymer. The improvement of flexural modulus is possible by abaca fiber reinforcement regardless of the fiber treatment. The further increase of flexural modulus is possible by surface etherification of the abaca fiber. The flexural strength did not increase even if BA-or AA-abaca fiber is used. [11] The flexural modulus was improved with increasing bamboo powder content when the cylinder temperature of the injection molded was 140degree Celsius The flexural strength of composite decreases with increasing bamboo powder content. Flexural strength was improved with increasing bamboo powder contents in biodegradable composite. The bamboo powder content was fixed at $60 \mathrm{wt} \%$. Higher flexural strength and modulus are obtained at (180 and 200) degree Celsius. The specimen surface prepared using injection molding at 200 degrees Celsius is more uneven than that prepared at 120degree Celsius. Higher flexural properties were obtained at higher temperature rather than lower temperature. It might be presumed that the interfacial adhesion strength between the bamboo fiber and PBS increased at higher cylinder temperatures. [12]

\section{Scanning Electron Microscopy}

[13] The dispersion state of GNSs in the PBS matrix was characterized by scanning electron microscopy and the SEM image of the fracture surface of PBS nanocomposite with $2.0 \mathrm{wt} \%$ GNS loading is shown. It can be clearly observed that grapheme sheets are uniformly dispersed in PBS matrix. SEM was performed on a Hitchi X650 (SEM) with acceleration voltage of $20 \mathrm{kw}$. Sample were prepared by immersing the film in liquid nitrogen for $10 \mathrm{~min}$ before fracture. [14] sem micrographs of NHAP/PLLA scaffolds fabricated using dioxide and water mixture solvent. SEM micrographs of plain PLLA, NHAP/PLLA and MHAP/PLLA scaffolds fabricated from 5\% dioxide (a-e) and benzene (fig) solution. Pure PLLA scaffold, 50, 400; MHAP/PLLA 50:50 scaffold, 100, 500; NHAP/PLLA 50:50 scaffold, 100, 1000; (g) Tubular NHAP/PLLA scaffold, crosssection, 200; (h) Tubular NHAP/PLLA scaffold, longitudinal section, 100.Mechanical Properties: The compressive modulus of NHAP/PLLA scaffolds increased with NHAP content .The plain PLLA scaffolds prepared using solid-liquid phase separation techniques had a compressive modulus of 4.3MPa.The modulus increased significantly when the NHAP proportion reached $30 \%$ of the composite and reached 8.3MPa when the ratio of NHAP to PLLA was 50:50.[15]SEM analysis shows that Tio 2 and pbs nanoparticles are anchored on the graphene sheets after sol-gel treatment. The Figure shows the image of PBS -Graphene/Tio2, which suggest that sheet morphology is retained even after the attachment of PBS and Tio2 nanoparticles on the graphene sheets. The layer interlayer spacing and thin layer edges of the graphene sheet can be clearly observed. The morphology of the graphene is observed having a flaky texture which reflects its layer structure. It is clearly seen that the Tio2 particles are aggregated are bind together by Vander walls forces in more loosely associated agglomerates fused together. This agglomerate will provide sites for absorbing fluids and will enhance the protocatalytic activity. The crystal structure and phases of the samples were obtained by XRD with Cu K (Alpha) radiation (Wavelength=1.54056) in the range of (10-80) degrees. The scan speed 1.2 (meter inverse). [16] PZT matrix is $\mathrm{Pb}(\mathrm{No} 3) 2$ and Na2s solution at room temperature. The XRD spectra shows the presence of tetragonal phase of PZT. The size of pbs at samples surface is about $25 \mathrm{~nm}$, Estimated by AFM Analysis. The roughness of the samples surface is about 5nm. The porosity of the composite estimated from the SEM analysis in cross-section is smaller than for the reference PZT porous matrix. The thickness of PBZ porous matrix is much larger than that of PBZ layer. SEM is not the optimum method of analysis because it has less resolution. The cross sectional SEM analysis also show very clearly the presence of quasi -continuous ultra-thin layer (about 50mm) on the top of PZT porous matrix with a characteristic grain size of 25mm. [17] An SEM photomicrographs of the fracture surface for bamboo and pbs composite showed typically fractured behavior of pull-out fibers without fiber fracture. There was no adhesion of PBS resin on bamboo fiber surface. Processing conditions affected mechanical properties of bamboo and PBS composite, imparting higher flexural strength and flexural modulus at high cylinder temperatures such as 180 Celsius and 200 Celsius. The figure shows that there are many pull-out fibers without fiber fractures and that no sticking of PBS resin exists on face of bamboo fibers. This result is likely to be derived from the low adhesive strength between the bamboo powder and PBS because the bamboo powder surface was untreated in the study. The cylindrical temperature is 140 Celsius the bamboo powder content is $40 \mathrm{wt}$. \%

\section{Impact strength}

[18] Izod impact strength presents the notched Izod impact strength of RHF (80-100 mesh) filled PBS, PP, and HDPE composites. The Izod impact strength of RHF-filled PBS bio composites was lower than that of RHF-filled PP and RHFfilled 
HDPE composites because of the very brittle characterization of PBS. The impact strength of bio composites is affected by the matrix polymer rather than by the addition of the filler in composites system. Therefore, we can compare the tensile and impact Figure 5 Notched Izod impact strength of RHF (80-100mesh) filled PBS, PP, and HDPE bio composites. PPRHF and HDPE-RHF. The notched Izod impact strength of the bio composites, shown at different filler loadings in decreased as the agro-flour content increased. Generally, notched impact strength is a measure of crack propagation. The poor interfacial adhesion between agro flour and the matrix polymer causes micro cracks when impact occurs, thus allowing the cracks to easily propagate. As the particle size increased, the notched Izod impact strength slightly increased. This result was expected because the crack propagates at the weaker RHF-PBS interface as well as through the biopolymer. Because of cracks travelling around the RHF particles, the fracture surface area increases with increasing particle size. Therefore, more energ $y$ is required to fracture the impact specimen with larger particles Stress - strain curves of agro-flour-filled PBS bio composites. For PBS-RHF at 0 strain :izod impact strength is 5, For PP-RHF at 0 strain :izod impact strength is 18 joules/m2,For HDPE-RHF at 0 strain :izod impact strength is 14.For PBS-RHF at 10 strain :izod impact strength is 4.7 kjoule/m2, For PP-RHF at 10 strain :izod impact strength is 16 joules/m2 For HDPE-RHF at 10 strain :izod impact strength is $14.1 \mathrm{kjoule} / \mathrm{m} 2$.For PBS-RHF at 40 strain :izod impact strength is 8 kjoule/m2, For PP-RHF at 40 strain :izod impact strength is $3.7 \mathrm{kjoule} / \mathrm{m} 2$,For HDPE-RHF at 40 strain :izod impact strength is 14 kjoule/m2. [19] Basalt Fiber Content (vol $\%$ ) the microstructure of the composite and dictates its mechanical properties, as the composite is stiffer and stronger in the direction of maximum orientation. Therefore, the orientation coefficient represents how the alignment of the fibers affects the fiber reinforcing effect and the flexural properties more sensitive to the fiber loading than the tensile ones. In order to determine the response of the composites to dynamic loading, the impact strength of the materials prepared was determined by notched Izod impact tests. The impact strength of the BF/PBS composite is not changed at a fiber loading of 3 vol\% because the lower loading of fibers does not work when the fracture occurs. The impact strength goes up almost linearly with increasing fiber loading between 5 and $15 \mathrm{vol} \%$. The highest value at the fiber loading of $15 \mathrm{vol} \%$ is $7.5 \mathrm{~kJ} \mathrm{~m}-2$. On impact, the energy of the pendulum is transferred to the test specimen and part of the energy is consumed during fracture. The total work-of-fracture can be calculated as the accumulative work of the different possible mechanisms to dissipate the energy, such as the work-on-fracture of the reinforcing fibers and the matrix respectively; the energy dissipated due to different fibermatrix interactions (sliding, debonding, fiber pullout, etc.). For notched specimens, the impact strength of the composites experimented drastically increases as the proportion of reinforcement fibers augmented. It can be inferred that basalt fibers play an effective role as reinforcement and improve the mechanical properties of PBS in the present system. [20] Impact in the thermal stability above 400_C was ascribed to the different content of lignin present in the polymer matrix. Figure 5 shows the impact and flexural strength results of different fiber reinforced polymer composites. It was observed that among all the fibers, sisal and curaua fibers demonstrated a huge potential as reinforcement in PBS matrix. This behavior was attributed to impact resistance results of different fiber-reinforced polymer composites. Impact resistance vs polymer composites. For P-bagasse impact strength is 80 (kilojoules per meter 2), For P-coconut impact strength is 90(kilojoules per meter 2), For P-curaua impact strength is 380(kilojoules per meter 2). For P-sisal impact strength is 560. [21]5mm of fiber length has $10.75 \mathrm{~kJ} / \mathrm{m}^{\wedge}$ square. $10 \mathrm{~mm}$ of fiber length has $11.75 \mathrm{~kJ} / \mathrm{m}^{\wedge}$ square. $15 \mathrm{~mm}$ of fiber length has $11 \mathrm{~kJ} / \mathrm{m}^{\wedge} \mathrm{square}$. $20 \mathrm{~mm}$ of fiber length has $9.75 \mathrm{~kJ} / \mathrm{m}^{\wedge}$ square. In addition, the incorporation of long fibers (in this case, fibers with lengths of 15 and $20 \mathrm{~mm}$ ) was also expected to resulting poor fiber dispersion and to promote. On unwanted fiber entanglement and, thus, resulted in inferior properties of the composites basis of these results, the PBS30KBF composite with 10-mm KBF was considered to be the best formulation for the PBS/KBF composite system. As seen in the composite with 10-mm KBF had $8.5 \%$ greater impact strength. In contrast, the composite with the 20 -mm fiber length had $8.6 \%$ poorer impact strength. The decrease in the impact strength could have been due to the severe fiber damage that resulted from composite compounding. [22] I impact strength of PBS, PBS/OPMF, and PBS/OPEFBF bio composites. For 10\% OPMF impact strength will be 275 $\mathrm{j} / \mathrm{m}$, for $20 \%$ OPMF impact strength will be $210 \mathrm{j} / \mathrm{m}, 30 \%$ OPMF impact strength will be $175 \mathrm{j} / \mathrm{m}, 40 \%$ OPMF impact strength will be $170 \mathrm{j} / \mathrm{m}, 70 \%$ OPMF impact strength will be $90 \mathrm{j} / \mathrm{m}$. For $10 \%$ OPEFBF impact strength will be $260 \mathrm{j} / \mathrm{m}$, for 20\%OPEFBF impact strength will be $160 \mathrm{j} / \mathrm{m}$. for 30\%OPEFBF impact strength will be $165 \mathrm{j} / \mathrm{m}$. For 40\%OPEFBF impact strength will be $110 \mathrm{j} / \mathrm{m}$. for $70 \%$ OPEFBF impact strength will be $60 \mathrm{j} / \mathrm{m}$. the impact strength of un-notched samples of PBS, PBS/OPMF, and PBS/OPEFBF bio composites. It is evident that the impact strength of PBS bio composites is lower than that of neat PBS regardless of type and content of fibers. PBS/OPMF bio composites show higher degree of impact strength as compared to those of PBS/OPEFBF bio composites. PBS is ductile or plastic like in nature, can absorb more energy during impact failure, and, hence, has high impact strength. However, the presence of OPMF or OPEFBF in the PBS matrix has turned the ductile behavior of PBS to brittle as indicates by decreasing of impact strength of about 33-84\% or 36-89\%, respectively. According to Mat Taib the introduction of stiff fiber in a ductile matrix will restrict the segmental motion of the polymer chains, which will consequently limit the deformability of the matrix phase, resulting in low impact strength.

\section{Conclusion}

The compression of tensile: strength will be $209.21,7 \mathrm{~N} 72(\mathrm{Mpa})$ treated :strength will be $228.54(\mathrm{Mpa})$ It is seen that at $5 \%$ alkali solution when soaking time increases from $24 \mathrm{~h}$ to $72 \mathrm{~h}$ the tensile properties of alkali-treated coir fibers increased, but they decreased beyond $72 \mathrm{~h}$. Based on standard methods of testing the flexural properties of rigid plastic (JIS K7203), Span length was $30 \mathrm{~mm}$, and the testing speed was $10 \mathrm{~mm}$ per min. the PBS matrix was characterized by scanning electron microscopy Pure PLLA scaffold NHAP/PLLA 50:50 scaffold, 100, 1000. Izod impact strength presents for P-sisal impact strength is $560 \mathrm{~kJ} / \mathrm{m}^{\wedge}$ square. 


\section{Reference}

[1].Kim, Hee-Soo, Han-Seung Yang, and Hyun-Joong Kim. "Biodegradability and mechanical properties of agro-flourfilled polybutylene succinate biocomposites." Journal of Applied Polymer Science 97, no. 4 (2005): 1513-1521. [2].Nam, Tran Huu, Shinji Ogihara, Nguyen Huy Tung, and Satoshi Kobayashi. "Effect of alkali treatment on interfacial and mechanical properties of coir fiber reinforced poly (butylene succinate) biodegradable composites." Composites Part B: Engineering 42, no. 6 (2011): 1648-1656.

[3].Zhang, Yihe, Chunxiao Yu, Paul K. Chu, Fengzhu Lv, Changan Zhang, Junhui Ji, Rui Zhang, and Heli Wang. "Mechanical and thermal properties of basalt fiber reinforced poly (butylenes succinate) composites." Materials Chemistry and Physics 133, no. 2-3 (2012): 845-849.

[4].Zhang, Yihe, Chunxiao Yu, Paul K. Chu, Fengzhu Lv, Changan Zhang, Junhui Ji, Rui Zhang, and Heli Wang. "Mechanical and thermal properties of basalt fiber reinforced poly (butylene succinate) composites." Materials Chemistry and Physics 133, no. 2-3 (2012): 845-849.

[5].Pulkit Purohit, M. Ramachandran, Selection of Flywheel Material using Multicriteria Decision Making Fuzzy Topsis, Indian Journal of Science and Technology, 8(33), 2015.

[6].Wang, Xin, Hongyu Yang, Lei Song, Yuan Hu, Weiyi Xing, and Hongdian Lu. "Morphology, mechanical and thermal properties of graphene-reinforced poly (butylene succinate) nanocomposites." Composites Science and Technology 72, no. 1 (2011): 1-6.

[7].Shibata, Mitsuhiro, Koichi Ozawa, Naozumi Teramoto, Ryutoku Yosomiya, and Hiroyuku Takeishi. "Biocomposites made from short abaca fiber and biodegradable polyesters." Macromolecular Materials and Engineering 288, no. 1 (2003): $35-43$.

[8].Nam, Tran Huu, Shinji Ogihara, Nguyen Huy Tung, and Satoshi Kobayashi. "Effect of alkali treatment on interfacial and mechanical properties of coir fiber reinforced poly (butylene succinate) biodegradable composites." Composites Part B: Engineering 42, no. 6 (2011): 1648-1656.

[9].Thakur, Vijay Kumar, Manju Kumari Thakur, and Raju Kumar Gupta. "raw natural fiber-based polymer composites." International Journal of Polymer Analysis and Characterization 19, no. 3 (2014): 256-271.

[10].Lee, Sang Muk, Donghwan Cho, Won Ho Park, Seung Goo Lee, Seong Ok Han, and Lawrence T. Drzal. "Novel silk/poly (butylene succinate) biocomposites: the effect of short fibre content on their mechanical and thermal properties." Composites Science and Technology 65, no. 3-4 (2005): 647-657.

[11].Nam, Tran Huu, Shinji Ogihara, Nguyen Huy Tung, and Satoshi Kobayashi. "Effect of alkali treatment on interfacial and mechanical properties of coir fiber reinforced poly (butylene succinate) biodegradable composites." Composites Part B: Engineering 42, no. 6 (2011): 1648-1656.

[12].Shibata, Mitsuhiro, Koichi Ozawa, Naozumi Teramoto, Ryutoku Yosomiya, and Hiroyuku Takeishi. "Biocomposites made from short abaca fiber and biodegradable polyesters." Macromolecular Materials and Engineering 288, no. 1 (2003): 35-43.

[13].Ohkita, Kazuya, and Hitoshi Takagi. "Flexural Properties of Injection-Molded Bamboo/pbs Composites." International Journal of Modern Physics B 24, no. 15 n16 (2010): 2838-2843.

[14].Wang, Xin, Hongyu Yang, Lei Song, Yuan Hu, Weiyi Xing, and Hongdian Lu. "Morphology, mechanical and thermal properties of graphene-reinforced poly (butylene succinate) nanocomposites." Composites Science and Technology 72 , no. 1 (2011): 1-6.

[15].Wei, Guobao, and Peter X. Ma. "Structure and properties of nano-hydroxyapatite/polymer composite scaffolds for bone tissue engineering." Biomaterials 25, no. 19 (2004): 4749-4757.

[16].Ullah, Kefayat, Ze-Da Meng, Shu Ye, Lei Zhu, and Won-Chun Oh. "Synthesis and characterization of novel PbSgraphene/TiO2 composite with enhanced photocatalytic activity." Journal of industrial and engineering chemistry 20, no. 3 (2014): 1035-1042.

[17].Stancu, V., M. Buda, L. Pintilie, M. Popescu, and F. Sava. "The ferroectric-electric characterization of PZT-PbS composites." Journal of optoelectronics and advanced materials 9, no. 5 (2007): 1516.

[18].Yan, Libo, Nawawi Chouw, and Xiaowen Yuan. "Improving the mechanical properties of natural fibre fabric reinforced epoxy composites by alkali treatment." Journal of Reinforced Plastics and Composites 31, no. 6 (2012): 425437. [19].Kim, Hee-Soo, Han-Seung Yang, and Hyun-Joong Kim. "Biodegradability and mechanical properties of agro-flour- filled polybutylene succinate biocomposites." Journal of Applied Polymer Science 97, no. 4 (2005): 15131521. [20].Zhang, Yihe, Chunxiao Yu, Paul K. Chu, Fengzhu Lv, Changan Zhang, Junhui Ji, Rui Zhang, and Heli Wang. "Mechanical and thermal properties of basalt fiber reinforced poly (butylene succinate) composites." Materials Chemistry and Physics 133, no. 2-3 (2012): 845-849.

[21].Thakur, Vijay Kumar, Manju Kumari Thakur, and Raju Kumar Gupta. "raw natural fiber-based polymer composites." International Journal of Polymer Analysis and Characterization 19, no. 3 (2014): 256-271.

[22].Thirmizir, MZ Ahmad, ZA Mohd Ishak, R. M. Taib, S. Rahim, and S. Mohamad Jani. "Kenaf-bast-fiber-filled biodegradable poly (butylene succinate) composites: Effects of fiber loading, fiber length, and maleated poly (butylene succinate) on the flexural and impact properties." Journal of Applied Polymer Science 122, no. 5 (2011): 3055-3063. [23].Then, Yoon Yee, Nor Azowa Ibrahim, Norhazlin Zainuddin, Hidayah Ariffin, and Wan Md Zin Wan Yunus. "Oil 
palm mesocarp fiber as new lignocellulosic material for fabrication of polymer/fiber biocomposites." International Journal of Polymer Science 2013 (2013).

[24].Vishal Fegade, Shannay Rawal, M.Ramchandran, Metamodel-based parametric study of composite laminates IOP Conf. Series: Materials Science and Engineering, 810(2020): 012051.

[25]. Joseph, Shibu, S. Jacob Melvin Boby, D. Muthu Gnana Theresa Nathan, and P. Sagayaraj. "Investigation on the role of cost effective cathode materials for fabrication of efficient DSSCs with TiNT/TiO2 nanocomposite photoanodes." Solar Energy Materials and Solar Cells 165 (2017): 72-81.

[26]. Vaithiyanathan, D., R. Seshasayanan, S. Anith, and K. Kunaraj. "A low-complexity DCT approximation for image compression with 14 additions only." In 2013 International Conference on Green Computing, Communication and Conservation of Energy (ICGCE), pp. 303-307. IEEE, 2013. 